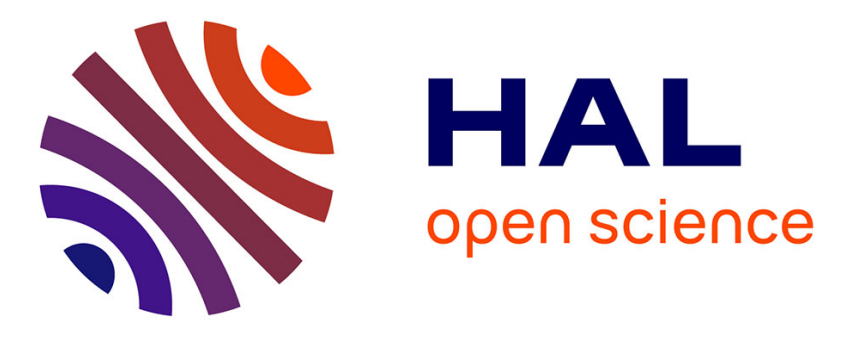

\title{
Deciphering the Dynamic Landscape of Transcription-Associated mRNP Quality Control Components Over the Whole Yeast Genome.
}

\author{
Kevin Moreau, Aurélia Le Dantec, A.R. Rahmouni
}

\section{- To cite this version:}

Kevin Moreau, Aurélia Le Dantec, A.R. Rahmouni. Deciphering the Dynamic Landscape of Transcription-Associated mRNP Quality Control Components Over the Whole Yeast Genome.. Methods in Molecular Biology, 2020, 2209, pp.251-265. 10.1007/978-1-0716-0935-4_16 . hal-03023042

\section{HAL Id: hal-03023042 \\ https://hal.science/hal-03023042}

Submitted on 25 Nov 2020

HAL is a multi-disciplinary open access archive for the deposit and dissemination of scientific research documents, whether they are published or not. The documents may come from teaching and research institutions in France or abroad, or from public or private research centers.
L'archive ouverte pluridisciplinaire HAL, est destinée au dépôt et à la diffusion de documents scientifiques de niveau recherche, publiés ou non, émanant des établissements d'enseignement et de recherche français ou étrangers, des laboratoires publics ou privés. 


\section{Chapter X}

Deciphering the dynamic landscape of transcription-associated mRNP quality control components over the whole yeast genome

Kévin Moreau, Aurélia Le Dantec and A. Rachid Rahmouni *

Centre de Biophysique Moléculaire, UPR 4301 du CNRS, 45071 Orléans, France

*Corresponding author: rahmouni@cnrs-orleans.fr

Running Head: Mapping mRNP QC components genome-wide in yeast 


\section{Abstract}

In eukaryotic cells, aberrant mRNPs with processing and packaging defects are targeted co-transcriptionally by a surveillance system that triggers their nuclear retention and ultimately the degradation of their mRNA component by the 3'-5' activity of the exosome-associated exonuclease Rrp6. This mRNP quality control process is stimulated by the NNS complex (Nrd1-Nab3-Sen1), which otherwise mediates termination, processing and decay of ncRNAs. The process involves also the exosome co-activator TRAMP complex (Trf4-Air2-Mtr4). Here, we describe a genome-wide approach to visualize the dynamic movement and coordination of these quality control components over the yeast chromosomes upon perturbation of mRNP biogenesis. The method provides valuable information on how the surveillance system is precisely coordinated both physically and functionally with the transcription machinery to detect the faulty events during perturbation of mRNP biogenesis. The overview shows also that the gathering of the quality control components over affected mRNA genes takes place at the expense of their commitment to be recruited at ncRNAs genomic features, provoking termination and processing defects of ncRNAs.

\section{Key Words:}

mRNP biogenesis; mRNP quality control; ncRNAs; yeast; RNA-seq; ChIP-seq 


\section{1- Introduction}

In the nucleus of eukaryotic cells, nascent pre-mRNA molecules are sequentially coated with a large set of processing and binding proteins that mediate their transformation into export-competent ribonucleoprotein particles (mRNPs) ready for translation in the cytoplasm. Genetic and biochemical studies, using the yeast Saccharomyces cerevisiae as a model organism, have suggested that the various mRNA processing and packaging reactions, known as mRNP biogenesis, are physically and functionally coupled to transcription elongation by RNA polymerase II (RNAP II). The co-transcriptional maturation and assembly of export-competent mRNPs is facilitated by the C-terminal domain (CTD) of the largest subunit of RNAP II that serves as a platform for sequential recruitment of various acting factors $[1,2]$. The production of mature export-competent mRNPs is also under the surveillance of quality control (QC) steps that are interconnected with transcription elongation and mRNP biogenesis. Defective mRNPs resulting from suboptimal processing and packaging reactions are targeted by the surveillance system leading to their retention in the nucleus with ensuing elimination of their mRNA component by the RNA degradation machinery [3-5].

A decade of extensive investigations using yeast mutants with defects in the THO-Sub2 complex, which mediates the assembly and export of a subset of mRNPs, allowed the identification of the nuclear degradation machinery involved in the mRNP QC process [6,7]. It was shown that the mechanism of nuclear retention and degradation of defective mRNPs depends on the presence of the nucleus specific exosome-associated exonuclease Rrp6 and its cofactor Rrp47 as well as components of the exosome activating complex TRAMP (Trf4-Air2-Mtr4). However, these analyses were unable to provide an integrated view of the QC system 
especially regarding the co-transcriptional recognition of mRNP aberrancies and its role in the subsequent triggering of mRNA decay. [Figure 1 near here]

The difficulty to decipher the actual mechanism of action underlying the QC process resides in the scarcity of mRNP biogenesis defects occurring under physiological context. In effect, the putative frequency of mRNP aberrancies occurring under physiological conditions does not allow a direct study of the QC mechanism within the cellular context. To circumvent this limitation, we recently implemented a new experimental approach based on global perturbation of mRNP biogenesis by the RNA-dependent helicase/translocase activity of the bacterial Rho factor. The heterologous expression of Rho in yeast induces a dose-dependent growth defect that stems from its interference in the co-transcriptional recruitment of mRNA processing and packaging factors yielding mRNPs that are recognized as aberrant and eliminated by the nuclear QC system [8]. In this approach, sufficient amounts of defective mRNPs can be produced by Rho action in the cell nucleus to serve as substrates for the QC, which facilitates deep investigations of the surveillance process [9]. Using this methodology, we have shown that the QC process is coordinated by Nrd1, a component of the NNS (Nrd1-Nab3-Sen1) complex known to mediate termination and processing of the non-coding RNAs (ncRNAs) and which is recruited by the CTD of RNAP II through its CTD-interacting domain (CID) [10]. The Rho-mediated production of aberrant mRNPs stimulates the recruitment of Nrd1 with its partner Nab3 to the transcription complex, which promotes the attachment of Rrp6 along with its co-activating components Rrp47 and Trf4 [11,12]. In this chapter, we provide a full description of our Rho-based experimental approach and outline its use to investigate the mRNP QC activities over the whole yeast genome. High-throughput RNA sequencing (RNA-seq) was used to survey a 
broad collection of mRNPs whose biogenesis is affected by Rho action (Figure 1).

This genome-wide perspective of affected mRNPs was extended by generating highresolution maps (ChIP-seq) of the distribution of the QC components along the yeast chromosomes before and after perturbation of mRNP biogenesis by Rho. As illustrated in Figure 2, the perturbation of mRNP biogenesis redistributes the binding of QC components over the genome with a large shift of Nrd1 and Nab3 from genomic loci producing ncRNAs prone to processing and decay such as CUTs (Cryptic Unstable Transcripts), SUTs (Stable Unannotated Transcripts) and snoRNAs (small nucleolar RNAs) to protein-coding genes. Nrd1 and Nab3 are apparently titrated out from ncRNAs genomic features by a large recruitment to Rho-affected mRNA gene loci, leading to transcriptional termination defects of ncRNAs [13]. This approach can be adapted to any protein potentially involved at the chromatin level in mRNA surveillance and/or processing. It can also be extended to the study of ncRNAs whose processing relies on the same machineries as mRNA.

[Figure 2 near here]

\section{2- Materials}

\subsection{Yeast strains, plasmids and growth media}

1. All Saccharomyces cerevisiae strains used for the present work are listed below. They are derived from the wild-type strain BMA41 (MATa or MATa ade2-1 ura3-1 leu2-3,112 his3-11,15 trp1 $\Delta$ can 1-100) [14] and were constructed by homologous recombination (see Note 1)

- $\quad \operatorname{rrp} 47 \Delta$, like BMA41 with $r r p 47 \Delta:: K A N$

- NAB3-MYC, like BMA41 with Nab3-MYC::HIS3

- NRD1-MYC, like BMA41 with Nrd1-MYC::HIS3 
- RRP6-MYC, like BMA41 with Rrp6-MYC::HIS3

- TRF4-MYC, like BMA41 with Trf4-MYC::HIS3

2. pCM185-Rho-NLS plasmid, a derivative of the pCM185 vector (CEN, TRP) from Euroscarf (http://www.euroscarf.de) expressing bacterial Rho factor in yeast with a C-terminal NLS (Nuclear Localization Signal) and under the control of a Doxycycline-regulated (Tet-off) promoter [8].

3. Liquid media for the growth of yeast cells, prepared from a mix of 3 different powders. For $900 \mathrm{ml}$ of media, weigh $18 \mathrm{~g}$ of glucose, $6.03 \mathrm{~g}$ of YNB (Yeast Nitrogen Base) containing ammonium sulfate and $0.67 \mathrm{~g}$ of CSM -Trp (Complete Synthetic Medium without Tryptophan) dissolved in $900 \mathrm{ml}$ of distilled water (see Note 2). After dissolution, dispatch into 3 portions of 300 $\mathrm{ml}$ in $500 \mathrm{ml}$ bottles and autoclave. Store the autoclaved media for up to 6 months at room temperature.

4. $1,000 x$ stock solution of Doxycycline at $5 \mathrm{mg} / \mathrm{ml}$. Dissolve $125 \mathrm{mg}$ of doxycycline in $25 \mathrm{ml}$ of $50 \%$ ethanol/water $(\mathrm{v} / \mathrm{v})$, store at $-20^{\circ} \mathrm{C}$. The Tet promoter is repressed by addition of doxycycline to the growth medium at a final concentration of $5 \mu \mathrm{g} / \mathrm{ml}$ just before use.

5. Specific instruments and labware:

- Precision balance for weighting the powder for media preparation

- Vortex for homogenization of solution

- Magnetic plate for agitation (with heating function)

- Centrifuge for pelleting the yeast cells when needed

- Glass or plastic flasks for cell growth

- Optical density measurement device to monitor cell growth

- Rotary shaker (250-300 rpm) for cell growth at controlled temperature. 


\subsection{RNA extraction solutions}

1. DEPC-treated water.

2. AE buffer: $50 \mathrm{mM}$ sodium acetate, $\mathrm{pH} 5.2,10 \mathrm{mM}$ EDTA

3. $10 \%(w / v)$ sodium dodecyl sulfate (SDS) solution in water.

4. Buffer-saturated, phenol:chloroform solutions, at $\mathrm{pH} 4.6$ and at $\mathrm{pH} 6.7$.

5. $3 \mathrm{M}$ sodium acetate solution, $\mathrm{pH} 5.2$.

6. Ethanol absolute (>99\%)

7. $75 \%$ ethanol in water.

8. Dry ice.

9. $2 \mathrm{U} / \mu \mathrm{L}$ RNase-free DNase I with $10 x$ supplied buffer.

10. Specific instruments and labware:

- Dedicated set of pipettes and special RNase free tips.

- Fume hood to avoid inhaling phenol:chloroform vapes.

- Nanodrop spectrophotometer or equivalent.

\subsection{Chromatin ImmunoPrecipitation (ChIP)}

1. TBS buffer: $20 \mathrm{mM}$ Tris- $\mathrm{HCl}, \mathrm{pH} 7.5,150 \mathrm{mM} \mathrm{NaCl}$.

2. 1X Phosphate buffered saline (PBS), $\mathrm{pH} 7.4$.

3. PBS/BSA mixture: dissolve $125 \mathrm{mg}$ Bovine Serum Albumin (BSA) in $25 \mathrm{ml} 1 \mathrm{X}$ PBS.

4. $0.1 \mathrm{M}$ PMSF solution. Dissolve $430 \mathrm{mg}$ of protease inhibitor Phenylmethylsulfonyl fluoride (PMSF) in $25 \mathrm{ml}$ of isopropanol and store as 1 $\mathrm{ml}$ aliquots at room temperature until use (see Note 3). 
5. $37 \%$ formaldehyde solution in water. We use a commercial solution from Sigma, which also contains $10-15 \%$ methanol as stabilizer.

6. $2.5 \mathrm{M}$ Glycine in water. Sterilize with a $0.22 \mu \mathrm{m}$ filter and store as aliquots at $20^{\circ} \mathrm{C}$.

7. $1 \mathrm{X}$ TE buffer: $10 \mathrm{mM}$ Tris- $\mathrm{HCl}$ and $1 \mathrm{mM}$ EDTA, $\mathrm{pH} 8$.

8. Lysis buffer 140: $50 \mathrm{mM}$ HEPES pH 7.5, $140 \mathrm{mM} \mathrm{NaCl}, 1 \mathrm{mM}$ EDTA, 1 \% (v/v) Triton X-100, $0.1 \%(\mathrm{w} / \mathrm{v})$ sodium deoxycholate. Right before use, add 1:100 volume of 0.1 M PMSF (see Note 4).

9. Lysis buffer 360: $50 \mathrm{mM}$ HEPES pH 7.5, $360 \mathrm{mM} \mathrm{NaCl}, 1 \mathrm{mM}$ EDTA, 1 \% (v/v) Triton X-100, $0.1 \%(\mathrm{w} / \mathrm{v})$ sodium deoxycholate. Right before use, add 1:100 volume of PMSF 0.1 M (see Note 4).

10. Washing buffer: $10 \mathrm{mM}$ Tris- $\mathrm{HCl} \mathrm{pH} \mathrm{8,} 0.25 \mathrm{M} \mathrm{LiCl,} 0.96 \%$ (v/v) NP-40, $0.5 \%$ (w/v) SDS, 1 mM EDTA.

11. Elution buffer: $50 \mathrm{mM}$ Tris-HCl pH 8, $10 \mathrm{mM}$ EDTA, $1 \%$ (w/v) SDS.

$12.10 \mathrm{mg} / \mathrm{ml}$ Proteinase $\mathrm{K}$ in water.

13. anti c-MYC sc-40X antibody at $200 \mu \mathrm{g} / \mathrm{ml}$ (Santa Cruz Biotechnology).

14. Protein G PLUS-agarose beads (Sigma).

15. Specific instruments and labware:

- Centrifuge with temperature control (must be maintained at $4^{\circ} \mathrm{C}$ ).

- Heating bloc.

- RNase/DNase free tips with barrier filter.

- Fastprep device (MB Biomedicals) for cell breakage or an equivalent apparatus.

- Quick PCR purification kit (see Note 5).

- Sonication apparatus (see Note 6). 


\subsection{Libraries preparations and NGS data treatment}

For the preparation of sequencing libraries from immunoprecipitated DNA fragments and inputs, different kits and specific tools are needed:

1. NEBNext Ultra II DNA Library Prep for Illumina from New England Biolabs or equivalent from other suppliers.

2. Qubit assay kit (Thermo Fisher Scientific)

3. Magnetic rack device for washing steps of the NEBNext Ultra II kit.

4. Magnetic beads (specific brands are suggested in the NEBNext Ultra II kit)

5. Spectrophotometer for Qubit assays measurement.

6. Academic core facility or commercial service for high-throughput nucleic acid sequencing, e.g. on an Illumina NextSeq 500 (see Note 7).

7. 64 GB RAM, 12 cores computer, equipped with Linux Ubuntu operating system and R software.

8. Software for NGS data treatment, e.g. as provided by the web-based Galaxy platform (https://usegalaxy.org/).

\section{3- Methods}

The first parts of each subsection are purely experimental and explain how we used Rho expression in yeast, how we extract RNA to perform high-throughput sequencing and how we perform chromatin immunoprecipitation to reveal the recruitment of QC 
components over the genome. The subsequent parts are dedicated to the use of bioinformatics to analyze the genome-wide sequencing results.

\subsection{Cell Growth and Rho-induction for perturbation of mRNP biogenesis.}

The expression of Rho in yeast is repressed by growing the cells in the presence of the tetracycline analogue, Doxycycline (Doxy), at a final concentration of $5 \mu \mathrm{g} / \mathrm{ml}$, whereas the omission of Doxy in the growth medium allows maximum expression. The cell growth is monitored by measuring the optical density at $600 \mathrm{~nm}\left(\mathrm{OD}_{600}\right)$.

1. Inoculate the S. cerevisiae strain of interest transformed by the plasmid pCM185-Rho-NLS (TRP1 Marker) from the $-80^{\circ} \mathrm{C}$ stock or from a streaking on a fresh plate into $5 \mathrm{ml}$ of liquid medium minus Tryptophan and with Doxy at a final concentration of $5 \mu \mathrm{g} / \mathrm{ml}$. Then, let the cells grow in a rotating incubator at $25^{\circ} \mathrm{C}$ overnight.

2. The following morning, use $800 \mu \mathrm{l}$ of the culture to inoculate $10 \mathrm{ml}$ of fresh medium containing $0.5 \mu \mathrm{g} / \mathrm{ml}$ of Doxy and grow the cells at $25^{\circ} \mathrm{C}$ for approximately 6 hours until an $O D_{600}$ of 0.5 .

3. Wash the cells three to four times with fresh medium lacking Doxy by sequential centrifugations at $3500 \mathrm{~g}$ for $5 \mathrm{~min}$ at $5{ }^{\circ} \mathrm{C}$ and removal of the supernatant (see Note 8).

4. Dilute the cells and allow them to grow overnight in $200 \mathrm{ml}$ flasks containing 40 to $45 \mathrm{ml}$ of the medium lacking Doxy to achieve maximum Rho expression. In parallel, grow similar cultures with medium containing $5 \mu \mathrm{g} / \mathrm{ml}$ Doxy for the non-induced samples. After an overnight growth (14-16 h), the Rho-induced 
cells are typically at an $\mathrm{OD}_{600}$ of $0.25-0.3$, whereas the non-induced samples are at an $\mathrm{OD}_{600}$ between 0.8 and 1 (see Note 9 ).

5. To analyse the samples, dilute the non-induced cultures with fresh medium to adjust the $\mathrm{OD}_{600}$ to the induced cultures before harvesting the cells (RNA extraction) or crosslinking (ChIP).

\subsection{RNA extraction}

Total RNA extraction is performed using the acidic hot phenol/chloroform method according to [15] with some modifications.

1. After adjusting the non-induced and induced cultures to the same lowest $\mathrm{OD}_{600}$, put back the yeast liquid cultures in the agitating incubator at $25^{\circ} \mathrm{C}$ for $20 \mathrm{~min}$.

2. During this 20 min meantime prepare all the necessary buffers and stand ready to perform all the following manipulations on ice.

3. Transfer the yeast cultures to $50 \mathrm{ml}$ tubes, then centrifuge at $4{ }^{\circ} \mathrm{C}, 10 \mathrm{~min}$, $3500 \mathrm{~g}$. Remove the supernatant and wash the pellet with $1 \mathrm{ml}$ of DEPCtreated water.

4. Re-suspend the cell pellet in $1 \mathrm{ml}$ of DEPC-treated water by vortexing and transfer the suspension to $1.5 \mathrm{ml}$ microtubes. Centrifuge the tubes in a benchcentrifuge at $4000 \mathrm{~g}$ for approximately one minute. Remove the supernatant and add $400 \mu \mathrm{l}$ of $\mathrm{AE}$ buffer and $40 \mu \mathrm{l}$ of $10 \%$ SDS, then vortex to suspend the cells. 
5. Transfer the suspensions into $1.5 \mathrm{~mL}$ microtubes previously filled with micro glass beads $(\sim 1 / 5$ of the tube). The next steps have to be performed under a fume hood.

6. Add an equivalent volume $(440 \mu \mathrm{l})$ of hot phenol/chloroform pH 4.5 (previously warmed up to $65^{\circ} \mathrm{C}$ ).

7. Vortex the tubes for $1 \mathrm{~min}$, then incubate them at $65^{\circ} \mathrm{C}$ for $1 \mathrm{~min}$. This is repeated 3 times to optimize the mechanical breakage of the cells.

8. Put the samples in ethanol/dry-ice bath for 2 min (alternatively, they can be stored 2 hours at $-80^{\circ} \mathrm{C}$ ). Put back the samples to the $65^{\circ} \mathrm{C}$ incubator for 3 min, vortex, and repeat the process twice.

9. Centrifuge the tubes at $4{ }^{\circ} \mathrm{C}, 12 \mathrm{~min}, 15000 \mathrm{~g}$ in a bench-centrifuge then carefully pipette the aqueous phase and transfer it in a new tube. After treatment with DNase I (as recommended by the supplier) to remove possible DNA contaminants, add an equivalent volume of phenol/chloroform $\mathrm{pH} 6.7$, vortex and centrifuge at $4{ }^{\circ} \mathrm{C}$ for $10 \mathrm{~min}$ at $13000 \mathrm{rpm}$.

10. Carefully pipet the aqueous phase and transfer it in a new tube, then add sodium acetate to a final concentration of $0.3 \mathrm{M}$. Add 3 volumes of $100 \%$ ethanol, and precipitate at $-80^{\circ} \mathrm{C}$ for two hours followed by centrifugation at $4^{\circ} \mathrm{C}$ for $20 \mathrm{~min}$ at $15000 \mathrm{~g}$. Remove the supernatant and wash the pellet with $75 \%$ ethanol. Dry the pellet briefly and resuspend it in $30 \mu \mathrm{l}$ of RNase-free water. Then, the RNA can be quantified from a $\sim 1 \mu \mathrm{L}$ aliquot with a Nanodrop (or equivalent) spectrophotometer (assuming an extinction coefficient at 260 $\mathrm{nm}$ for RNA of $\left.0.025(\mu \mathrm{g} / \mathrm{ml})^{-1} \mathrm{~cm}^{-1}\right)$.

\subsection{RNA-seq libraries preparation and sequencing}


cDNA libraries preparations from isolated RNA were made by I2BC high-throughput sequencing platform (Gif sur Yvette) using Illumina ScriptSeq protocol after RiboZero treatment and followed with sequencing on an Illumina NextSeq 500 instrument. We always outsource the preparation of RNA-seq libraries to NGS platforms because they usually have high expertise and are better equipped for the task, especially regarding the quality checks of the samples. For each condition (Rho-repressing or Rho-inducing conditions), two biologically independent samples are used for libraries preparation and sequencing.

\subsection{RNA-seq data processing and computational analysis}

In this section, we provide an overview of the bioinformatics packages and manipulations used for the sequencing analysis and data processing pipeline (see Note 10):

1. Run a quality control analysis of the reads using FastQC $v 0.11 .5$ package.

2. Remove the adaptor's sequences from the reads using Cutadapt 1.15.

3. Use the bwa 0.7.12 software package to map the reads on the reference genome (Saccharomyces cerevisiae genome V64.1.1). The genome annotations can be downloaded from SGD (https://www.yeastgenome.org/) and specific ncRNAs annotations are taken from [16] for SUTs and from [17] for CUTs.

4. Quality control the alignment to the genome with Samtools 0.1.19.

5. Process the data by counting the number of reads per genomic feature using the package featureCounts V 1.5.0-p1, with -s 1 option. 
6. Isolate a working pool of mRNAs from the Wt strain that are affected by Rho action by comparing the mRNA levels between the - Rho and + Rho conditions. This determines which mRNAs from the data sets of the Wt strain are becoming down-regulated under Rho action. For these differential expression analyses use DESeq2 package under R environment (V 3.4.4).

7. Perform the same differential analysis on the isolated mRNA pool using the data sets collected from the rrp47 $\Delta$ strain, which lacks the exonuclease Rrp6.

8. Using the two sets of results obtained above (Wt and $\operatorname{rrp} 47 \Delta$ strain), sort the mRNAs by their sensitivity to Rho action and which can be rescued under Rrp6 depletion (Figure 1A).

9. To visualize the effect of Rho as reads coverage, use the same subsets of the reads results obtained for the selected pools of mRNAs to generate Metatranscript profiles variations of mRNAs between $\mathrm{Wt}$ and the rrp47D strain in the absence or presence of Rho (Figure 1B). To perform these analyses, generate BigWig files using bedtools V2.25.0 (genomecov and bigwigCompare), and UCSC tool bedGraphToBigWig. After computing genome coverage with genomecov, convert the bedgraph outputs to bigwig files using bedGraphToBigWig. Meta-profiles plots are then made using bedtools 24 V2.25.0 with computeMatrix (scale-regions mode, with a bin size of 1 base) on BigWig files and plotProfile for graphical output.

\subsection{Chromatin ImmunoPrecipitation (ChIP)}


Each protein of interest within the cross-linked chromatin complex is immunoprecipitated via the MYC tag present at its C-terminus using an antibody recognizing the MYC epitope (anti C-MYC sc-40X antibody).

1. At the end of the overnight growth, as for RNA extraction, adjust the $O D_{600}$ of the non-induced cells by dilution with medium in order to obtain the same number of cells in each culture/condition.

2. To perform protein-nucleic acids crosslinking within the chromatin add $1.2 \mathrm{ml}$ of $37 \%$ formaldehyde solution to the $\sim 45 \mathrm{ml}$ of yeast culture (from Section 3.1 ) directly into the culture flask and incubate for $20 \mathrm{~min}$ at room temperature on a rotating platform within a fume hood.

3. Stop the cross-linking reaction by adding $2.25 \mathrm{ml}$ of $2.5 \mathrm{M}$ Glycine and incubate $5 \mathrm{~min}$ at room temperature, then put the samples on ice (see Note 11).

4. Transfer the culture into a $50 \mathrm{ml}$ conical tube and wash the cells three times with cold TBS by applying centrifugation steps at $3500 \mathrm{~g}$ for 5 min at $4{ }^{\circ} \mathrm{C}$ and carefully removing all supernatant (see Note 12)

5. Resuspend the washed cell pellet in $1 \mathrm{ml}$ of lysis buffer 140 by pipetting up and down and add the cell suspension directly to a $2 \mathrm{ml}$ screw cap microtube, containing glass-beads (same volume of beads than the cell suspension).

6. Break the cells with a Fastprep device in a cold room (4 cycles of $30 \mathrm{sec}$ at $6000 \mathrm{rpm}$ with a $1 \mathrm{~min}$ pause between each cycle).

7. To recover the cell extract without the glass-beads, drill a hole in the bottom of the microtube with an incandescent needle, then introduce it into a $15 \mathrm{ml}$ conical tube. After removing the screw cap, centrifuge $1 \mathrm{~min}$ at $1500 \mathrm{~g}$ to recover the extract. 
8. After recovering the extract and transferring it to a $2 \mathrm{ml}$ microtube, adjust the volume to $1.2 \mathrm{ml}$ with Lysis buffer 140 , then shear the cross-linked chromatin by sonication on ice to reduce average DNA fragments to an approximate size of $\sim 400-500$ base pairs (see Note 13).

9. Centrifuge the samples for $5 \mathrm{~min}$ at $5000 \mathrm{rpm}$ and $4{ }^{\circ} \mathrm{C}$ then recover the supernatant (approximately $1 \mathrm{ml}$ ) and put it in a new microtube.

10. Take $20 \mu \mathrm{l}$ of supernatant genomic DNA which will be used as Input sample and mix the remaining part with $4 \mu \mathrm{L}$ anti c-MYC antibody solution for the IP sample. Fixe the tubes on a rotating plate and incubate overnight at $4{ }^{\circ} \mathrm{C}$.

11. The next morning, add $37 \mu \mathrm{L}$ of the protein G PLUS-agarose beads slurry and let the samples rotate for 2 hours at $4{ }^{\circ} \mathrm{C}$.

12. Perform successive washing steps by $2-3$ min centrifugation at $4000 \mathrm{~g}$ and supernatant elimination at $4{ }^{\circ} \mathrm{C}$ (the agarose beads are pulled at the bottom of the tube): 2 times with $1 \mathrm{ml}$ lysis buffer 140, 2 times with $1 \mathrm{ml}$ lysis buffer 360 , 1 time with $1 \mathrm{ml}$ washing buffer and 1 time with $1 \mathrm{ml} \mathrm{TE}$.

13. Elute the DNA from the agarose beads twice with $75 \mu$ l of elution buffer during 10 min incubations at $65^{\circ} \mathrm{C}$, each time recovering supernatant.

14. The samples ( $150 \mu \mathrm{l}$ of IP and the $20 \mu \mathrm{l}$ of Input adjusted to $150 \mu$ l by addition of elution buffer) are reverse crosslinked by incubation at $65^{\circ} \mathrm{C}$ overnight.

15. Add to each sample $15 \mu \mathrm{l}$ of $10 \mathrm{mg} / \mathrm{ml}$ proteinase $\mathrm{K}$ and incubate for digestion $1.5 \mathrm{~h}$ at $37^{\circ} \mathrm{C}$. Recover the DNA samples using a PCR Purification Kit (see Note 5), following manufacturer's instructions.

\subsection{DNA libraries preparations and sequencing for ChIP-seq}


ChIP-seq libraries preparations in duplicates are done following the NEBNext Ultra II DNA Library Prep kit for Illumina from New Englands Biolabs as outlined by the supplier. Briefly, the kit workflow can be divided in three major steps (see Note 14).

1. The first step is applied directly on the DNA fragments from the ChIP samples and is based on the enzymatic preparation of both ends of the DNA fragments.

2. The second step consists of adaptors ligation at each end.

3. The third step uses the ligated adaptors as a platform for hybridization of primers (possessing unique barcodes used for multiplexed sequencing) and subsequent amplification of the DNA fragments by PCR.

4. A Qubit assay is then performed following the manufacturer's instructions to evaluate the amount of DNA obtained at the end of the PCR amplification (see Note 14).

5. The libraries are sent to the pre-selected high-throughput sequencing platform that runs an Illumina NextSeq 500.

\subsection{ChIP-seq data analysis}

Upon completion of the high throughput sequencing, download the results and perform the data analyses according to the following steps (see Note 15):

1. Run a quality control of the reads using FastQC $v 0.11 .5$ package.

2. Process the trimming of the adaptor's sequences from the reads using Cutadapt 1.15 package.

3. Map the whole reads on the reference Saccharomyces cerevisiae genome (version V64.1.1) with the bwa 0.7.12 software package. 
4. Quality control the alignment of the reads to the reference genome using Samtools 0.1.19 package.

5. Proceed to a specific peak-calling step using the PePr V1.1.21 package by calculating reads ratios between IP and Input in a comparative mode where only peak signals detected simultaneously in the two replicates are considered. The piled-up peak signals obtained for the perturbed condition (+Rho) and non-perturbed condition (-Rho) are subsequently subjected to a step of differential analysis where only peaks appearing for one condition but not the other are scored (see Note 16).

\section{4- Notes}

1. Each strain carrying a C-terminally MYC-tagged protein for the ChIP experiments was constructed by integration of appropriate DNA fragments encoding the Tag at the protein gene locus following homologous recombination. The DNA fragments were obtained by PCR amplification with appropriate primers on plasmid template pYM19 [18]. The strains can be obtained from us upon request. Alternatively, similar strains derived from the wild-type background strain BY4741 are available from the Euroscarf yeast collection (http://www.euroscarf.de/).

2. The synthetic complete medium is without Tryptophan to maintain within the yeast cells the centromeric plasmid pCM185-Rho-NLS that has the TRP1 marker.

3. Stock solutions of PMSF in $100 \%$ isopropanol do not need any sterilization and they are stable for at least 9 months at 15 to $25^{\circ} \mathrm{C}$. 
4. Upon addition of 1:100 volume of $0.1 \mathrm{M} \mathrm{PMSF}$, the final working concentration of PMSF is $1 \mathrm{mM}$.

5. We use the GeneJET PCR purification kit (ThermoFischer) but other brands such as Quiaquick PCR purification kit (Qiagen) work as well.

6. Depending on what is available, use either a probe sonicator device while maintaining the sample on ice or use a water-bath sonicator such as the BIORUPTOR (Diagenode), which has the advantage of keeping the samples at $4{ }^{\circ} \mathrm{C}$ during sonication.

7. We recommend to have an early contact with the NGS outsourcing platform to discuss the specific needs (single end reads or paired reads, number of reads per library), the experimental design and the schedule for the sequencing operations.

8. Cell washing is an important step to remove all the residual Doxy and thus to allow full expression of Rho during growth for the induced samples.

9. The difference of the growth density between the cells grown under repressing conditions and those grown under inducing conditions results from the growth defect induced by Rho expression.

10. All the bioinformatics data processing packages are freely available and can be downloaded from respective web sites and run with Linux Ubuntu operating system. Alternatively, the Galaxy webserver (https://usegalaxy.org/) which is under a graphical user interface can also be used for data processing. But this alternative shows less flexibility in terms of option and data manipulations.

11. From this step, try to work on ice so the samples are kept as close as possible to $4^{\circ} \mathrm{C}$. 
12. If necessary for timing purpose, the processing of the samples can be stopped at this step by storing the cell pellets at $-80^{\circ} \mathrm{C}$.

13. As specified in Note 6, the shearing of the chromatin can be done with a BIORUPTOR or a usual sonicator with a slim probe that fits into a $2 \mathrm{ml}$ tube. With our sonicator device (Autotune Model 750 watts), we use the following parameters: $20 \%$ amplitude, 7 sec of pulse ON and 3 sec of pulse OFF for a total time of 4 min with the probe diving at 2/3 inside the microtube which is maintained on ice. These parameters should be calibrated for each specific sonication device by analysing the size of the DNA fragments on agarose gel.

14. It is good to know that this kit can be used for a broad range of initial DNA quantity (from $500 \mathrm{pg}$ to $1 \mu \mathrm{g}$ ). Another advice when using this kit is to avoid numerous PCR amplification cycles as far as possible in order to circumvent PCR duplicates that can make analysis bias during bioinformatics processing of the sequencing data.

15. All the bioinformatics data processing packages are freely available and can be downloaded from respective web sites. Note that the data processing of the ChIP-seq results can also be run on the Galaxy webserver (https://usegalaxy.org/) as for RNA-seq.

16. Peak calling using the PePr V1.1.21 package allows the analysis of duplicates directly and in a comparative mode when needed. This differential analysis reveals clearly Rho-mediated shifts of recruitment and thus, it gives a snapshot of the dynamic landscape of the QC components. Manipulations on peak caller outputs are done mostly within $R$ environment (V 3.4.4). Figures are built with ggplot2 package and Circos plots are created using OmicCircos package. All snapshots used for direct visualization of reads coverage or ChIP 
peaks distributions were taken with the IGV browser (Integrative Genomics Viewer). 


\section{Legends to Figures}

Figure 1: Fate of subpopulations of mRNAs down-regulated by Rho action in yeast wild-type strain $(\mathrm{Wt})$ and their rescue in a strain depleted of the exonuclease Rrp6 (rrp474). (A) Violin plots showing a significant drop in mRNAs levels in Wt strain under Rho perturbation (cyan plot) and the rescue of a subpopulation levels in the mutant strain (pink plot). For each genetic background (WT or rrp474), duplicates from Rho-induced conditions (+Rho) were compared to duplicates from Rho repressed conditions (-Rho). The statistically down-regulated mRNAs ( $p$-value $<0.05$ and L2FC $<-0.5)$ from WT background in +Rho conditions $(1,015$ mRNAs) were isolated and their relative levels were computed in the mutant (rrp474) conditions which showed a pool of 491 mRNAs that were rescued. (B) Meta-transcript profiles variations of mRNAs obtained from Wt and the Rrp6 depleted rrp47 4 strain in the absence or presence of Rho. The top graph represents the down-regulated mRNAs subpopulation in the presence of Rho in Wt cells. The bottom graph displays the meta-transcript profiles of the pool of mRNAs down-regulated by Rho action in Wt strain and which were rescued upon Rrp6 depletion (rrp47A strain). After computing genome coverage (with genomecov), bedgraph outputs were converted to bigwig files using bedGraphToBigWig. Meta-profiles plots were made using bedtools 24 V2.25.0 with computeMatrix (scale-regions mode, with a bin size of 1 base) on BigWig files and plotProfile for graphical output. Student $t$ test (bidirectional) was used to compare levels of Log2 Fold Change L2FC between RNA-seq conditions. NS $>0.05>^{*}>0.01>^{* *}>0.001>^{* * *}$.

Figure 2: Rho activity mediates the stimulation of massive recruitment of the QC components (Nrd1, Nab3, Rrp6 and Trf4) to rrp6-sensitive mRNAs genomic loci at 
the expense of their locations within ncRNAs genomic features. (A) IGV snapshots of ChIP-seq peaks mapped over two rescued mRNAs genomic loci. The ChIP signals for each protein are shown both for - Rho and + Rho conditions. (B) Circos plots summarizing the observed dynamic landscape of the four QC components over the 16 yeast chromosomes analyzed under -Rho (left plots) and + Rho (right plots) conditions. The two top plots show the ChIP signals detected for the ncRNAs genomic loci (CUTs, SUTs and snoRNAs). The two plots at the bottom show the ChIP signals detected for the genomic features of the Rho-affected mRNAs that were rescued by Rrp6 depletion. The red arrow symbolizes the hijacking of Nrd1 and Nab3 from ncRNAs genomic loci to the Rho-affected mRNA genes upon perturbation of mRNP biogenesis by Rho. Student $t$ test (bidirectional) was used to compare Log2 average ChIP-seq signals. 


\section{References}

1. Bentley DL (2014) Coupling mRNA processing with transcription in time and space. Nat Rev Genet 15, 163-175

2. Luna R, Gaillard H, Gonzalez-Aguilera C et al. (2008) Biogenesis of mRNPs: integrating different processes in the eukaryotic nucleus. Chromosoma 117, 319-331

3. Doma MK, Parker R (2007) RNA quality control in eukaryotes. Cell 131, 660-668

4. Fasken MB, Corbett AH (2005) Process or perish: quality control in mRNA biogenesis. Nat Struct Mol Biol 12, 482-488

5. Schmid M, Jensen TH (2013) Transcription-associated quality control of mRNP. Biochim Biophys Acta 1829, 158-168

6. Rougemaille M, Gudipati RK, Olesen JR et al. (2007) Dissecting mechanisms of nuclear mRNA surveillance in THO/sub2 complex mutants. EMBO J 26, 2317-2326

7. Villa T, Rougemaille M, Libri D (2008) Nuclear quality control of RNA polymerase II ribonucleoproteins in yeast: Tilting the balance to shape the transcriptome. Biochim Biophys Acta 1779, 524-531

8. Mosrin-Huaman C, Honorine R, Rahmouni AR (2009) Expression of bacterial Rho factor in yeast identifies new factors involved in the functional interplay between transcription and mRNP biogenesis. Mol Cell Biol 29, 4033-4044

9. Mosrin-Huaman C, Hervouet-Coste, N., Le Dantec, A., Stuparevic, I., Rahmouni, A.R. (2014) Bacterial Rho helicase: a new tool to dissect mRNP biogenesis and quality control in yeast. Trends in Cell \& Molecular Biology 9, 79-93

10. Vasiljeva L, Kim M, Mutschler H et al. (2008) The Nrd1-Nab3-Sen1 termination complex interacts with the Ser5-phosphorylated RNA polymerase II C-terminal domain. Nat Struct Mol Biol 15, 795-804 
11. Honorine R, Mosrin-Huaman C, Hervouet-Coste N et al. (2011) Nuclear mRNA quality control in yeast is mediated by Nrd1 co-transcriptional recruitment, as revealed by the targeting of Rho-induced aberrant transcripts. Nucleic Acids Res 39, 2809-2820

12. Stuparevic I, Mosrin-Huaman C, Hervouet-Coste N et al. (2013) Cotranscriptional recruitment of RNA exosome cofactors Rrp47p and Mpp6p and two distinct Trf-Air-Mtr4 polyadenylation (TRAMP) complexes assists the exonuclease Rrp6p in the targeting and degradation of an aberrant messenger ribonucleoprotein particle (mRNP) in yeast. J Biol Chem 288, 31816-31829

13. Moreau K, Le Dantec A, Mosrin-Huaman C et al. (2019) Perturbation of mRNP biogenesis reveals a dynamic landscape of the Rrp6-dependent surveillance machinery trafficking along the yeast genome. RNA Biology 16, 879-889

14. Baudin-Baillieu A, Guillemet E, Cullin C et al. (1997) Construction of a yeast strain deleted for the TRP1 promoter and coding region that enhances the efficiency of the polymerase chain reaction-disruption method. Yeast 13, 353-356

15. Schmitt ME, Brown TA, Trumpower BL (1990) A rapid and simple method for preparation of RNA from Saccharomyces cerevisiae. Nucleic Acids Res 18, 3091-3092

16. Xu Z, Wei W, Gagneur J et al. (2009) Bidirectional promoters generate pervasive transcription in yeast. Nature 457, 1033-1037

17. Neil H, Malabat C, d'Aubenton-Carafa Y et al. (2009) Widespread bidirectional promoters are the major source of cryptic transcripts in yeast. Nature 457, 1038

18. Janke C, Magiera MM, Rathfelder N et al. (2004) A versatile toolbox for PCR-based tagging of yeast genes: new fluorescent proteins, more markers and promoter substitution cassettes. Yeast $\mathbf{2 1}, \mathbf{9 4 7 - 9 6 2}$ 